\title{
La Evaluación Formativa en el Proceso Enseñanza-aprendizaje en Estudiantes de Actividad Deportiva de la Universidad de Costa Rica
}

\author{
Formative Assessment in the Learning-teaching Process \\ with Sporting Activity Students at the University of Costa Rica \\ Jessenia Hernández Elizondo ${ }^{a}$, Alejandro Salicetti Fonseca ${ }^{b}$ \\ ${ }^{\mathrm{a}}$ Universidad de Costa Rica \\ Correo electrónico: jessenia.hernandez@ucr.ac.cr \\ ${ }^{\mathrm{b}}$ Universidad de Costa Rica \\ Correo electrónico: alejandro.salicetti@ucr.ac.cr
}

\section{RESUMEN}

La evaluación formativa favorece el proceso de enseñanza aprendizaje en el estudiantado y la interacción profesor alumno. El objetivo principal del presente trabajo fue emplear la evaluación formativa para valorar los aprendizajes por parte del estudiantado que participa de la clase de actividad deportiva. Ochenta estudiantes universitarios contestaron un cuestionario de autoreporte diseñado para dicho fin de forma voluntaria. Se encontró una valoración positiva en el desarrollo socioafectivo, físicomotor y cognitivo del estudiantado al asistir a los cursos de actividad deportiva. Se concluye que los beneficios percibidos por parte del estudiantado reflejan la importancia de estos cursos dentro del currículo universitario del ciclo formativo. Se recomienda aumentar en al menos un ciclo lectivo más la matrícula de los cursos de actividad deportiva, ya que esto puede potenciar los beneficios percibidos.

Palabras claves: evaluación formativa, educación superior, actividad física, salud.

\footnotetext{
ABSTRACT

Formative assessment promotes the learning process in the student and interaction between student and teacher. The main objective of this work was to employ formative assessment to determine the impact of learning for students participating in a sporting activity course. Eighty university students answered a self-reporting questionnaire designed for this purpose on a voluntary basis. A positive assessment of the socio-affective, physical-motor, and cognitive development of the students attending the course was noted. We conclude that the benefits perceived by students reflect the importance of such courses within the university curriculum. We recommend an increase of at least one additional school year of enrollment in sporting activity courses, as this may enhance the benefits noted.

Keywords: formative assessment, higher education, physical activity, health.
} 


\section{INTRODUCCIÓN}

Tomando en cuenta su reconocido paradigma humanista, la Universidad de Costa Rica crea, a partir de 1957, la Escuela de Estudios Generales, cuyo fin ha sido contribuir a la formación de personas íntegras, creativas, críticas, solidarias y con los más altos valores humanísticos (Arrieta \& Díaz, 1989). Para esto, se ofrece al estudiantado en sus primeros años de formación distintas opciones en las diversas áreas académicas. Las escuelas, que contribuyen con esta misión de la Universidad, ofrecen cursos de servicio para todas las carreras: los Seminarios de Realidad Nacional, cursos de Repertorio, cursos de Arte y cursos de Actividad Deportiva.

Desde sus orígenes, la Escuela de Educación Física y Deportes de la Universidad de Costa Rica no solo ha tenido como principal objetivo formar profesionales en el área de las ciencias del movimiento humano, sino que, desde 1957, fruto de las gestiones del profesor Rodrigo Leiva (de origen chileno) entonces director, se aprobó impartir regularmente cursos de actividad deportiva obligatorios dentro del ciclo de estudios generales como anteriormente mencionamos, manteniéndose hasta el presente como una de las actividades principales de la unidad académica (García, 1979).

Los cursos de actividad deportiva buscan brindar a la población estudiantil universitaria la oportunidad de formar y potenciar los hábitos para la práctica de la actividad física, con la finalidad de que puedan integrarlos a la vida diaria y así contribuir con estilos de vida saludable para mejorar la calidad de vida. Los cursos son de modalidad práctica, se imparten en dos sesiones de clases continuas de 50 minutos cada una de ellas y tienen los siguientes objetivos generales:

- Estimular el desarrollo de los canales socioafectivo, físicomotor y cognitivo de los estudiantes mediante la participación activa en las lecciones.

- Adquirir conocimientos y destrezas que permitan a los estudiantes integrar en su vida, la práctica de la actividad física continua como parte del mantenimiento y el desarrollo de su salud integral.

Con respecto a la evaluación, los cursos de actividad deportiva se aprueban con la asistencia y participación activa: realizar todas las actividades organizadas por el profesor durante las clases en todas las sesiones de clase. Esta forma de evaluación se enfoca en el proceso que vive el estudiante durante las clases prácticas. Según Casanova (2007), la evaluación formativa valora el proceso de enseñanza aprendizaje que el estudiante va adquiriendo en el desarrollo del curso y tiene como fin la retroalimentación continua para un mejor aprovechamiento. La evaluación del trabajo realizado en la clase es una de las formas en que se aplica la evaluación formativa.

Para los cursos de actividad deportiva, este proceso de evaluación formativa, dirigida a la evaluación de participación activa, se describe en la Tabla 1. 
Tabla 1. Proceso de Evaluación Formativa diario en los Cursos de Actividad Deportiva

\begin{tabular}{|l|l|l|}
\hline \multicolumn{1}{|c|}{ Momento de la clase } & \multicolumn{1}{|c|}{ Referente Teórico } & \multicolumn{1}{c|}{ Acciones } \\
\hline \multirow{2}{*}{ Metas a alcanzar } & Compartir metas de aprendizaje. & $\begin{array}{l}\text { En cada clase se desarrolla un } \\
\text { contenido para cumplir con un } \\
\text { objetivo propuesto. }\end{array}$ \\
\cline { 2 - 3 } & Clarificar criterios de logro. & $\begin{array}{l}\text { Se comparten las posibilidades de } \\
\text { alcance de logro de acuerdo al nivel } \\
\text { mostrado por los estudiantes. }\end{array}$ \\
\hline \multirow{2}{*}{ Estado actual } & Recolectar evidencias. & $\begin{array}{l}\text { Observación, registro de } \\
\text { información. }\end{array}$ \\
\cline { 2 - 3 } & Interpretar evidencia e identificar \\
la brecha de aprendizaje. & $\begin{array}{l}\text { Se compara con otros criterios. } \\
\text { Se compara el progreso del } \\
\text { estudiante. } \\
\text { La interpretación siempre considera } \\
\text { la opinión del estudiante. }\end{array}$ \\
\hline \multirow{2}{*}{ Cómo seguir mejorando? } & Ajustar la enseñanza. & $\begin{array}{l}\text { El profesor de acuerdo a las } \\
\text { experiencias es quien ajusta las } \\
\text { enseñanzas. }\end{array}$ \\
\cline { 2 - 3 } & Retroalimentación al estudiante. & Durante y al final de cada clase. \\
\hline
\end{tabular}

Después de realizado este proceso formativo en donde nuestros estudiantes y nuestros profesores evalúan constantemente sus avances con relación a los objetivos, con el propósito de lograr un aprendizaje significativo según los fines humanistas definidos por la Universidad, se propone evaluar la opinión de los estudiantes en relación al logro de los objetivos de la actividad deportiva del currículum universitario.

\subsection{OBJETIVOS DE INVESTIGACIÓN}

1. Emplear la evaluación formativa en el proceso de enseñanza aprendizaje en el estudiantado que participa de la clase de actividad deportiva.

2. Valorar los aprendizajes del estudiantado que participa de la clase de actividad deportiva.

\section{METODOLOGÍA}

\subsection{TIPO DE ESTUDIO}

Este estudio presenta una metodología multi-método (o mixta), la cual muestra ambos enfoques: el cualitativo y el cuantitativo. En proporciones similares, los métodos son concurrentes, sin embargo, existe un solapamiento de los sucesos porque se producen en intervalos de tiempo superpuesto vinculados en diferentes etapas de la investigación. Lo anterior permite un abordaje del tema con mayor profundidad (Hernández, Fernández \& Baptista, 2006). 


\subsection{PARTICIPANTES}

La muestra corresponde a 80 estudiantes, de tres cursos diferentes de actividad deportiva de la Escuela de Educación Física y Deportes de la Universidad de Costa Rica. La muestra utilizada fue de tipo intencional, así que todos los elementos de la población fueron seleccionados bajo estricto juicio de los investigadores. Los cursos fueron seleccionados por conveniencia: Actividades Aeróbicas Principiantes Mixto, Danza Aeróbica y Entrenamiento contra resistencia principiantes. La muestra final presentó un promedio de edad de $18.62 \pm 2.17$ años, se entrevistó a 48 mujeres y 32 hombres, de diferentes carreras universitarias.

\subsection{INSTRUMENTOS}

Para recolectar la información de los participantes se confeccionó un cuestionario de autoreporte. El cual fue validado previamente por el juicio de expertos, de tres profesionales en el área de Ciencias del Movimiento Humano, quienes son profesores de los cursos de Actividad Deportiva de la Escuela de Educación Física y Deportes.

El instrumento cuenta con un total de 12 preguntas, tanto de respuesta cerrada como de respuesta abierta (ver Tabla 2). Las preguntas de respuesta cerrada son de selección múltiple o dicotómica.

Tabla 2. Ítems del cuestionario y su escala

\begin{tabular}{|l|l|}
\hline \multicolumn{2}{|c|}{ Ítems } \\
\hline 1. & Sexo (respuesta dicotómica) \\
\hline 2. & Edad (años cumplidos) \\
\hline 3. & Carrera que estudia, año de la carrera \\
\hline 4. & ¿Por qué matriculó esta Actividad Deportiva? (selección múltiple) \\
\hline 5. & $\begin{array}{l}\text { ¿Favorecen las actividades realizadas la interacción alumno-alumno y la interacción } \\
\text { profesor-alumno? (respuesta abierta) }\end{array}$ \\
\hline 6. & $\begin{array}{l}\text { ¿Cómo se sintió al participar en la Actividad Deportiva? (escala Lickert de respuesta de } \\
6 \text { sentimientos; y opción de respuesta abierta) }\end{array}$ \\
\hline 7. & $\begin{array}{l}\text { ¿De qué forma cree usted que la Actividad Deportiva influyó en su condición física? } \\
\text { (respuesta abierta) }\end{array}$ \\
\hline 8. & $\begin{array}{l}\text { ¿Cree usted que la Actividad Deportiva influyó en su rendimiento académico? ¿Cómo? } \\
\text { (respuesta abierta) }\end{array}$ \\
\hline 9. & $\begin{array}{l}\text { Adquirió conocimiento de nuevas destrezas en las clases de Actividad Deportiva } \\
\text { (respuesta abierta) }\end{array}$ \\
\hline 10. & $\begin{array}{l}\text { Cómo puede poner en práctica las destrezas aprendidas en la Actividad Deportiva en su } \\
\text { vida cotidiana (respuesta abierta) }\end{array}$ \\
\hline 11. & $\begin{array}{l}\text { Su participación en la Actividad Deportiva le ha estimulado a realizar actividad física o } \\
\text { deporte como parte de su vida diaria (respuesta abierta) }\end{array}$ \\
\hline 12 & Le gustaría volver a matricular este u otro tipo de Actividad Deportiva (respuesta abierta) \\
\hline
\end{tabular}




\subsection{PROCEDIMIENTOS}

El primer paso de la investigación fue diseñar el cuestionario. En un grupo de trabajo compuesto por cinco especialistas en Ciencias del Movimiento Humano se confeccionaron las preguntas relacionadas con el impacto de la actividad deportiva en los diferentes dominios (socioafectivo, físicomotor y cognitivo). Una vez desarrolladas las preguntas se les entregó el cuestionario a tres evaluadores, también profesionales en el área. Los evaluadores debían contestar " 1 " si estaban de acuerdo con la pregunta o " 0 " si no estaban de acuerdo, y realizar una sugerencia; para así obtener un índice de concordancia entre evaluadores (CVR). Se modificaron las preguntas que presentaron un $\mathrm{CVR}<0.99$, lo que representa una concordancia menor a tres evaluadores. Posteriormente el grupo de trabajo, revisó las sugerencias y ajustó las preguntas. Para finalizar, se redactaron las preguntas de información personal y se les entregó nuevamente el cuestionario a los tres evaluadores para sus comentarios finales. En esta etapa se obtuvo un CRV = 0.99 para cada pregunta (Wilson, Pan \& Schumsky, 2012). Por lo tanto, el cuestionario presenta validez de contenido, dada por el juicio de expertos.

Para la aplicación del cuestionario se seleccionaron 80 estudiantes por conveniencia, pertenecientes a tres cursos de Actividad Deportiva. Se le solicitó permiso al profesor encargado para aplicar el cuestionario la penúltima clase del curso lectivo, al finalizar la sesión de clase. A la población estudiantil se le comentó el objetivo del cuestionario y se les solicitó de forma voluntaria completar el cuestionario. El estudiante que estuvo de acuerdo en ser parte del estudio contestó las preguntas de forma individual.

\subsection{ANÁLISIS DE LOS DATOS}

Los datos recolectados por medio del cuestionario fueron analizados de forma cualitativa o cuantitativa según la característica de la variable. Los datos cuantitativos se resumieron utilizando técnicas de estadística descriptiva (frecuencias, promedios, desviación estándar) con el programa de Microsoft Excel 2010. Los datos cualitativos se analizaron por medio de técnicas específicas del enfoque (análisis de contenido).

\section{RESULTADOS}

La muestra final consistió de 80 estudiantes universitarios, los cuales completaron el cuestionario de forma voluntaria. En la Tabla 3 se presenta la información de las preguntas 1 y 3 . 
Estudios Pedagógicos XLIV, $\mathrm{N}^{\circ}$ 2: 297-310, 2018

LA EVALUACIÓN FORMATIVA EN EL PROCESO ENSEÑANZA-APRENDIZAJE EN ESTUDIANTES DE ACTIVIDAD DEPORTIVA DE LA UNIVERSIDAD DE COSTA RICA

Tabla 3. Frecuencias de las características de los estudiantes encuestados

\begin{tabular}{|l|c|c|}
\hline \multirow{2}{*}{ Característica Generales } & \multicolumn{2}{|c|}{ Frecuencias } \\
\cline { 2 - 3 } & $\mathrm{n}$ & $\%$ \\
\hline Sexo & & \\
\hline Masculino & 32 & 40,00 \\
\hline Femenino & 48 & 60,00 \\
\hline Carrera por áreas & & \\
\hline Artes y letras & 7 & 8,75 \\
\hline Salud & 5 & 6,25 \\
\hline Ingeniería & 26 & 32,50 \\
\hline Ciencias Agroalimentarias & 3 & 3,75 \\
\hline Ciencias Básicas & 4 & 5,00 \\
\hline Ciencias Sociales & 34 & 42,50 \\
\hline No lo reportó & 1 & 1,25 \\
\hline Año de carrera & & \\
\hline Primero & 61 & 76,25 \\
\hline Segundo & 8 & 10,00 \\
\hline Tercero & 3 & 3,75 \\
\hline Cuarto & 4 & 5,00 \\
\hline Quinto-Sexto & 3 & 3,75 \\
\hline No lo reportó & 1 & 1,25 \\
\hline
\end{tabular}

Nota. Los valores representan frecuencias absolutas y relativas.

En la pregunta 4 ¿Por qué matriculó esta Actividad Deportiva? Según se muestra en la Tabla 4, la razón que anotan con mayor frecuencia las personas participantes es sentirse a gusto con la actividad deportiva que se ofrece, sumado a la conveniencia de horario que ese curso de actividad deportiva representa para ellos. Asimismo, las personas participantes agregaron otras razones, entre ellas: el deseo de aprender actividades diferentes, no tener la opción de matricular el deporte que más le gusta porque no se ofreció dentro de las opciones posibles, no saber de qué se trataba la actividad deportiva, pero tener la facilidad de que el horario se ajustaba a las necesidades, ajuste entre los horarios ofrecidos para cursar la actividad deportiva y la disponibilidad de tiempo de los estudiantes para matricular el curso, ser la actividad deportiva que les llamaba la atención o ser la opción disponible en el momento de la matrícula. Algunos comentarios específicos son: "quería algo diferente" (sujeto 67), "no había atletismo" (sujeto 11), "no estaba seguro de qué era, pero se ajustaba a mi horario" (sujeto 12), "se ajusta al horario" (sujeto 78), "por rellenar horario" (sujeto 54), "era lo que quedaba" (sujeto 23), "solo esa quedaba” (sujeto 80), "era la única que me llamaba la atención” (sujeto 3). 
Tabla 4. Pregunta 4 ¿Por qué matriculó esta Actividad Deportiva?

\begin{tabular}{|l|c|c|}
\hline \multirow{2}{*}{ Opciones de Respuesta } & \multicolumn{2}{|c|}{ Frecuencias } \\
\cline { 2 - 3 } & $\mathrm{n}$ & $\%$ \\
\hline Era la única que me gustaba y se ajustaba bien con mi horario. & 58 & 72,50 \\
\hline No me gustaba, pero era la que mejor se ajustaba con mi horario. & 10 & 12,50 \\
\hline Otros & 12 & 15,00 \\
\hline
\end{tabular}

Nota. Los valores representan frecuencias absolutas y relativas.

Con respecto a la pregunta 5, ¿Favorecen las actividades realizadas la interacción alumnoalumno y la interacción profesor-alumno?, el 100\% de los participantes respondió que sí. Con respecto a la interacción alumno-alumno, se obtuvo que el uso de actividades como juegos y el uso de estrategia de organización como, por ejemplo, el trabajo en grupo, el trabajo en parejas o tríos, favorecen la comunicación e interacción durante las diferentes lecciones. No menos importante, los estudiantes mencionan que en la actividad deportiva se realizan actividades que califican como entretenidas, divertidas, fuera de rutina que permiten crear un ambiente de confianza en el que pueden comunicarse, hablar, reír, conocer gente nueva e incluso hacer nuevos amigos. Algunos de los comentarios específicos son los siguientes:

"Sí, se comparte y se conoce a gente nueva" (sujeto 2), "sí, jugando con diversión, fuera de rutina" (sujeto 5), "sí a través de todas las actividades en equipo" (sujeto 6), "sí porque hay juegos que son grupales, lo que motiva la interacción" (sujeto 9), "sí me parece que toda la clase formamos un buen equipo, creo que todos venimos a divertirnos y compartimos mucho" (sujeto 18), "sí, porque al ser trabajos en equipo se llega a una amistad con los compañeros" (sujeto 30), "sí claro las personas pierden un poco de vergüenza y se unen más al trabajo en equipo" (sujeto 38), "sílas favorecen. Existen dinámicas grupales y trabajo en parejas o tríos que propician las relaciones sociales" (sujeto 53).

Basados en las respuestas de los estudiantes, se extrae también información sobre la interacción profesor-alumno, detectando que la interacción entre el profesor y el alumno se presenta en el momento en que el profesor brinda las indicaciones de las actividades por realizar o cuando corrige la ejecución de algunos ejercicios. Asimismo, se evidencia que los estudiantes perciben que pueden acercarse al profesor, conversar con él, y con esto lograr que haya respeto por las diferencias individuales. Es importante recalcar que los estudiantes perciben que el profesor no da órdenes, sino que se comunica cordialmente. Algunas respuestas específicas:

"sí, porque el profesor no solo da órdenes, sino que se relaciona y uno conoce a los compañeros" (sujeto 19), "sí claro, la mayoría de las actividades son en equipo, ya sea parejas o más, y la interacción profesor-alumno también, el profesor sí explica bien y es flexible ante situaciones de los alumnos" (sujeto 29), "sí, porque uno puede conversar con el profesor" (sujeto 42), "sí porque el profesor explica cómo usar las máquinas 
Estudios Pedagógicos XLIV, $\mathrm{N}^{\circ}$ 2: 297-310, 2018

LA EVALUACIÓN FORMATIVA EN EL PROCESO ENSEÑANZA-APRENDIZAJE EN ESTUDIANTES DE ACTIVIDAD DEPORTIVA DE LA UNIVERSIDAD DE COSTA RICA

individualmente y cómo hacer los ejercicios" (sujeto 45), "sí, ya que normalmente en las lecciones se trabaja en distintas parejas, lo que favorece las relaciones entre compañeros y con el profesor igualmente se mantiene una interacción” (sujeto 56).

A partir de las respuestas de los estudiantes, se obtiene también que el espacio de comunicación e interacción que brinda la actividad deportiva contribuye al desarrollo integral, ya que como lo expresa el sujeto 31 "Definitivamente, el deporte favorece la formación integral de las personas en todas las áreas: física, emocional, social, etc.”. Otro estudiante mencionó: "Sí, porque ayuda al desarrollo integral del estudiante" (sujeto 52), "Claro, ya que se ejecuta un movimiento corporal completo lo que favorece a todo el organismo" (sujeto 26).

En la Tabla 5 se presenta la información relacionada a la pregunta 6, en donde se le preguntó al estudiantado sobre sus sentimientos al participar durante la clase.

Tabla 5. Pregunta 6 ¿Cómo se sintió al participar de la Actividad Deportiva?

\begin{tabular}{|l|c|c|c|c|c|c|c|c|c|c|c|c|}
\hline \multirow{2}{*}{ Sentimiento } & \multicolumn{2}{|c|}{ Nada } & \multicolumn{2}{|c|}{ Un poco } & \multicolumn{2}{c|}{$\begin{array}{c}\text { Moderada- } \\
\text { mente }\end{array}$} & \multicolumn{2}{c|}{ Bastante } & \multicolumn{2}{l|}{ Muchísimo } & \multicolumn{2}{l|}{ No reportó } \\
\cline { 2 - 16 } & $\mathrm{n}$ & $\%$ & $\mathrm{n}$ & $\%$ & $\mathrm{n}$ & $\%$ & $\mathrm{n}$ & $\%$ & $\mathrm{n}$ & $\%$ & $\mathrm{n}$ & $\%$ \\
\hline Feliz & 0 & 0,00 & 2 & 2,50 & 15 & 18,75 & 33 & 41,25 & 30 & 37,50 & 0 & 0,00 \\
\hline Triste & 72 & 90,00 & 4 & 5,00 & 1 & 1,25 & 0 & 0,00 & 0 & 0,00 & 3 & 3,75 \\
\hline Estresado & 60 & 75,00 & 12 & 15,00 & 3 & 3,75 & 1 & 1,25 & 1 & 1,25 & 3 & 3,75 \\
\hline A gusto & 0 & 0,00 & 1 & 1,25 & 5 & 6,25 & 35 & 43,75 & 36 & 45,00 & 3 & 3,75 \\
\hline Enojado & 72 & 90,00 & 3 & 3,75 & 2 & 2,50 & 0 & 0,00 & 0 & 0,00 & 3 & 3,75 \\
\hline Relajado & 4 & 5,00 & 6 & 7,50 & 24 & 30,00 & 20 & 25,00 & 23 & 28,75 & 3 & 3,75 \\
\hline
\end{tabular}

Nota. Los valores representan frecuencias absolutas y relativas.

Las personas participantes indicaron en comentarios adicionales que el sentimiento reportado se debe principalmente a que la actividad deportiva les brinda un espacio para hacer actividad física y eso es bueno para su salud, sobre todo porque en algunos casos sus actividades regulares no incluyen hacer actividad física. También mencionan que las actividades realizadas les ayudan a liberar el estrés que les ocasionan las tareas y obligaciones de ser un estudiante universitario.

Los adjetivos utilizados con mayor frecuencia para describir los tipos de actividades realizadas que les brindan sentimientos positivos (feliz, a gusto, relajado) incluyen las actividades son bonitas, excelentes, emocionantes, me recuerdan a mi niñez, me motivan a hacer actividad física, me ayudan a despejarme, estoy aprendiendo de los deportes, entre otros. Algunos de los comentarios específicos indicados son

"Demasiado divertidas, que sea en mecanismo de juegos" (sujeto 9), "la clase ayuda a despejarse del día a día de la universidad” (sujeto 15), "es una clase amena, la 
interacción entre todo el grupo es fenomenal, lo más agradable es que no nos aburrimos y aprendemos técnicas de juegos" (sujeto 18).

Por el contrario, las frases comunes que pueden predisponer a la persona durante la clase o estar asociadas a sentimientos negativos (triste, estresado, enojado) son el horario es incómodo, el baloncesto no me gusta, algunas actividades son muy competitivas y no me gusta, hay muchos juegos diferentes que se pueden implementar en la clase, entre otros.

En relación con la pregunta 7, las personas participantes indican comentarios donde se resalta un beneficio en la condición física. Este beneficio está evidenciado con comentarios similares a estos: siento más aire cuando hago las actividades, tengo más energía durante el día, sólo hago estas actividades una vez a la semana y esto me ha ayudado a mejorar y mantener mi condición física, antes me cansaba mucho haciendo cualquier actividad física, ahora resisto más, entre otros. Cabe destacar que muchos de los estudiantes indican que es la única actividad que hacen durante la semana, y aun así perciben que es suficiente para mejorar, ya que mencionan que no estaban acostumbrados a hacer ningún tipo de ejercicio. Además, indican que no se sienten sedentarios al hacer actividad una vez por semana.

"Es la única actividad física que realizo, entonces me ayuda a no ser sedentaria, mi condición ha mejorado" (sujeto 62), "influyó bastante porque me siento más activa y no tengo mucho cansancio durante el día” (sujeto 49).

Por el contrario, algunos estudiantes indican que la actividad deportiva influyó poco o nada en su condición física, y justifican su respuesta indicando que al ser solamente una vez por semana es poco ejercicio. Es probable que estos estudiantes sean físicamente activos y por eso no sientan una mejoría. Ya que como se mencionó anteriormente, las personas que no estaban acostumbradas a realizar ningún tipo de actividad física sí perciben una mejora. Ejemplos de estos comentarios son:

“no mucho porque solo practicaba en la clase y no el resto de los días" (sujeto 61), "poco, una vez a la semana es poco ejercicio" (sujeto 44).

Además, a los comentarios sobre la condición física las personas participantes indican que sienten mejoras en la resistencia muscular, ya que indican que se sienten más fuertes, que han desarrollado más músculo y tienen mayor resistencia en las piernas y brazos. También mencionan beneficios en aspectos psicológicos, como la motivación, la disciplina y disposición a realizar actividad física. Algunos comentarios son:

"me motivó a realizar deporte fuera de la universidad" (sujeto 25), "me matriculé en un gimnasio para hacer ejercicio otros días” (sujeto 33).

Con respecto a la pregunta 8 referida a si la actividad deportiva influyó en su rendimiento académico, una parte de los estudiantes indican que no influyó y otra parte expresó que sí. Se puede destacar que dentro de las opiniones de los estudiantes se mencionan otros beneficios asociados al buen rendimiento académico, como sentirse menos estresados frente a las demandas académicas, mejora en la disposición y energía para estudiar y asistencia a clases. 
Estudios Pedagógicos XLIV, $\mathrm{N}^{\circ}$ 2: 297-310, 2018

LA EVALUACIÓN FORMATIVA EN EL PROCESO ENSEÑANZA-APRENDIZAJE EN ESTUDIANTES DE ACTIVIDAD

DEPORTIVA DE LA UNIVERSIDAD DE COSTA RICA

En la pregunta 9 se les cuestionó si adquirieron conocimiento de nuevas destrezas en las clases de Actividad Deportiva y la totalidad de ellos contestaron afirmativamente. Los aprendizajes expresados por los estudiantes se pueden clasificar en tres categorías:

La primera categoría hace referencia al aprendizaje de nuevos ejercicios, tanto aeróbicos como de fuerza, orientados a mejorar el acondicionamiento físico y motor. Los estudiantes indican que el hacer ejercicio les contribuye al buen estado de la salud, les ayuda a sentirse mejor y con más vitalidad. Mencionaron, de manera específica, que aprendieron nuevos ejercicios que les ayudaban a mejorar la coordinación, agilidad y la flexibilidad.

Algunos de los comentarios expresados por los estudiantes para la categoría 1 fueron

"Aprendí nuevas maneras para ejercitarme en casa" (sujeto 1), "sobre nuevos pasos de aeróbicos” (sujeto 2).

“aprendí la técnica correcta de algunos ejercicios con máquinas" (sujeto 35) y

"el uso correcto de las pesas y las ligas" (sujeto 22).

En la categoría, 2 "Aprendizajes de deportes tradicionales y no tradicionales", la mayoría de los estudiantes resalta que los deportes resultaron para ellos divertidos, expresaron que aprendieron sobre deportes como

"El hockey, y carreras de relevo (sujeto 40), nuevos deportes (sujeto 19) y los deportes tradicionales como "el voleibol" (sujeto 13) y "el baloncesto" (sujeto 28).

Para el caso de la categoría 3, relacionada con que la práctica del ejercicio mejora los componentes psíquico, mental y social, los estudiantes indicaron que durante las clases de actividad deportiva el ejercicio y las distintas actividades físico-deportivas logran beneficios a nivel cognitivo y social. Algunos comentarios que anotaron los estudiantes fueron:

“Comprendí que por medio del deporte puedo mejorar la concentración” (sujeto 25). "me di cuenta que el deporte estimula el trabajo en equipo y ayuda a desarrollar destrezas interpersonales” (sujeto 31).

Con respecto a las respuestas de la pregunta 10 que indica ¿cómo puede poner en práctica las destrezas aprendidas en la Actividad Deportiva en su vida cotidiana?, en los comentarios de los estudiantes se aprecia que fueron fuertemente potenciados en la posibilidad de que ellos mismos construyan sus rutinas de ejercicio para realizar en casa, de manera individual o en grupo, según lo prefieran. Muchos fueron los comentarios que los estudiantes expresaron en esta línea. Por otro lado, algunos de los estudiantes manifestaron la posibilidad de matricularse en algún gimnasio privado o contratar un entrenador personal. Un ejemplo de los comentarios es el siguiente:

"Después de determinar el curso puedo crear yo misma una coreografía y repetirla" (sujeto 06).

Al preguntarle a los estudiantes si la participación en la actividad deportiva le ha estimulado a realizar actividad física o deporte como parte de su vida diaria (pregunta 11), la mayoría menciona que después de terminada la asignatura tienen más claro la 
importancia de la actividad física para la salud, consideran importante que realizar una actividad física moderada con regularidad es una de las maneras más sencillas de mejorar su salud y mantenerse sanos. Por otro lado, los estudiantes, al finalizar la asignatura, consideran también que el mantener actividad física de manera regular puede generar cambios muy positivos en sus vidas.

"Me estimula a no ser tan sedentaria y a ejercitarme, es la única actividad física que hago, pero intentaré el próximo semestre hacer más ejercicio" (sujeto 2).

Otro de los aspectos importantes que mencionan los estudiantes es el relacionado con las actividades programadas por los docentes. Ellos indican que las actividades propuestas por los profesores durante las clases los motivó a participar activamente.

“Las actividades que nos puso el profesor eran muy divertidas” (sujeto 40).

Uno de los aspectos a destacar en las respuestas de los estudiantes es el referido al tiempo para realizar ejercicio, algunos estudiantes indican que no pueden realizar actividad física por falta de tiempo, principalmente justificado por las exigencias de los estudios y el cansancio que éste representa y que algunos trabajan mientras estudian. Sin embargo, algunos estudiantes indican que actualmente ya tienen incorporado dentro de sus actividades cotidianas actividades físicas.

"Toda la vida he practicado deporte" (sujeto 10), "no tengo tiempo, ya que trabajo y estudio" (sujeto 56).

Sobre la pregunta 12 , donde se menciona si les gustaría volver a matricular este u otro tipo de actividad deportiva, la gran mayoría de los estudiantes, luego de finalizada la asignatura, indican que estarían dispuestos a matricular nuevamente la asignatura de actividad deportiva. Por el contrario, son muy pocos los estudiantes quienes luego de finalizada la asignatura indican que no estarían dispuestos a matricular la asignatura de actividad deportiva.

"Sí me encantaría llevar de nuevo el curso, pues es muy divertido y me ayudo a lograr un mejor desarrollo de las destrezas, y eso me ayuda con mi salud" (sujeto 20), "por cuestiones de tiempo y prefiero ejercitarme en momentos de conveniencia” (sujeto 2).

En resumen, las respuestas de la mayoría de los estudiantes brindaron un panorama positivo en relación con el aprovechamiento de los cursos de actividad deportiva. Se visualiza un proceso de enseñanza-aprendizaje en donde el estudiantado aprovechó las sesiones de manera satisfactoria para favorecer el aprendizaje de las áreas socioafectiva, fisicomotor y cognitiva, ya que en general expresaron que pudieron integrar los conocimientos y destrezas adquiridas en sus actividades cotidianas para promover un estilo de vida físicamente activo. 


\section{DISCUSIÓN}

Utilizar la evaluación formativa favorece el proceso de aprendizaje en el estudiantado y la interacción profesor-alumno, además de impulsar la comprensión, reflexión y aplicación de la aprendido (Hortigüela, Pérez-Pueyo \& López-Pastor, 2015; Romero-Martín, CastejónOliva y López-Pastor, 2015). En el presente estudio se analizó este tipo de evaluación en el curso de actividad deportiva dentro del currículo universitario de la Universidad de Costa Rica. Sin embargo, como indican Hortigüela et al. (2015) el uso de esta metodología debe ser comprobado, para así destacar si la percepción del profesor y del estudiantado en relación con su uso es positiva, y de esta manera justificar su aplicación.

Los resultados obtenidos en el presente estudio sugieren que el estudiantado se siente a gusto, feliz y relajado durante la clase de actividad deportiva. Estos sentimientos positivos favorecen el proceso de aprendizaje y la interacción con el profesor. Es importante destacar que el rol del docente es un factor fundamental en el proceso, ya que este es el encargado de brindar retroalimentación eficiente, que facilite la comprensión del contenido, garantizando el aprendizaje del mismo (Hortigüela et al., 2015). En estos cursos al ser presenciales, facilita el contacto continuo con el profesor, lo que potencia el proceso de aprendizaje debido a la disponibilidad del mismo para aclarar dudas.

Asimismo, el hecho de que los estudiantes perciban un ambiente en el que se pueden mantener abiertos canales de comunicación tanto con los compañeros como con el profesor, facilita la participación activa, lo cual fomenta la adquisición de un aprendizaje significativo y profundo (González-Peiteado \& Pino-Juste, 2014), que a su vez proporciona el hecho de que la persona encuentre una aplicación práctica de lo aprendido (Bain, 2007), lo que le permitirá aplicar e incluir en su vida cotidiana la actividad física.

La información obtenida sobre los aprendizajes demuestra que la participación activa por parte de los estudiantes los faculta en el conocimiento de nuevas formas para ejercitarse y en la adquisición y perfeccionamiento de destrezas por medio de diferentes deportes y/o actividades físicas, que motivan al estudiante a continuar un estilo de vida activo. Este aporte lo confirma Renzi (2009), quien expresa que la práctica de actividad física fortalece el desarrollo de capacidades coordinativas y condicionales y mejora los modos de conducta promoviendo una consciencia del movimiento más eficiente.

Aunque los participantes reconocen los beneficios físicos, psíquicos y sociales que la actividad físico-deportiva produce, los estudiantes de la universidad tienden a abandonar la práctica deportiva justificando que no tienen tiempo porque la demanda académica es mucha y además algunos tienen que trabajar para poder continuar estudiando. Estos resultados obtenidos concuerdan con Martínez y otros (2012), quienes luego de analizar el motivo del abandono a la práctica deportiva en 2859 estudiantes universitarios indican que son la exigencia del estudio y la pereza lo que suponen las principales causas de abandono. El conocer estos motivos nos sugiere mantener estos cursos de actividad deportiva y a la vez hacerlos más eficaces y duraderos con el objetivo que los estudiantes adopten o mantengan estilos de vida saludables. Aun cuando muchos estudiantes ya tenían incorporado en su vida cotidiana actividades físicas como caminar, andar en bicicleta o asistir frecuentemente a programas de ejercicios.

Además, los resultados del presente estudio concuerdan con los de una investigación meta-analítica donde se menciona que las clases de educación física presentan un impacto positivo en resultados relacionados con rendimiento académico $(T E=1.03$ ) (Watson et 
al., 2017). No obstante, menos de la mitad de los estudiantes indica que no percibieron beneficios, esto puede ser debido a que la duración de las actividades deportivas es relativamente corta, una hora y media a la semana durante el semestre universitario. Sin embargo, una cuarta parte de los estudiantes comentó que sí les benefició en componentes cognitivos como la concentración y el ir a clases o a estudiar con menos estrés.

Al tomar en cuenta que los cursos de actividad deportiva se evalúan mediante la participación activa de los estudiantes, las actividades que proponen los profesores durante las clases plantean una serie de estrategias y orientaciones para tratar de aumentar la integración de los estudiantes durante las clases prácticas. Algunas de las estrategias de enseñanza que utilizan los profesores con el fin de promover aprendizajes significativos están relacionadas con los siguientes elementos:

1. Aspectos de organización como la planificación anticipada de las actividades, introducción y explicación de actividades y selección de actividades con propuestas de variantes.

2. Aspectos motivacionales y actitudinales como implicar al alumno en la selección de los contenidos, creación de actitudes que promuevan la participación y el trabajo por objetivos a corto-mediano plazo.

3. Aspectos metodológicos como la selección de contenidos para conseguir clases más dinámicas, adecuado espacio de trabajo de acuerdo al número de participantes y la intención de igualar niveles de destrezas.

El uso de la evaluación formativa en los cursos de actividad deportiva para valorar los aprendizajes de la participación en clase de los estudiantes fue útil al evidenciar en ellos nuevas formas para ejercitarse y los beneficios que este conlleva. Concuerdan nuestros resultados obtenidos con lo mencionado por (Asún-Dieste, Romero-Martín \& ChiviteIzco, 2017), quienes reportan beneficio al utilizar la evaluación formativa para potenciar el aprendizaje del estudiantado. Bajo este punto de vista, la evaluación formativa de los estudiantes durante las clases podría funcionar como un buen proceso de enseñanza aprendizaje, ya que brinda la oportunidad de acompañar al estudiante durante las actividades propuestas por los profesores, dándonos cuenta de lo que ellos han aprendido día a día y ofreciéndonos a su vez mucha información sobre el proceso de adquisición de aprendizajes o saberes.

\section{CONCLUSIONES Y RECOMENDACIONES}

En función de los dos objetivos planteados en el presente estudio, la aplicación de la evaluación formativa durante el desarrollo del curso de actividad deportiva resultó ser de gran provecho, ya que permite potenciar de una mejor manera los aprendizajes que cada estudiante va logrando. En general, permite valorar, durante el proceso, el crecimiento del estudiantado y a su vez dar una guía personal.

En cuanto a la valoración de los aprendizajes, se encontró un aprovechamiento significativo, pues la mayoría de los estudiantes expresan beneficios físicos, cognitivos, sociales y emocionales. No obstante, una minoría manifestó que la asistencia a la actividad deportiva le limita el tiempo disponible para otras actividades de índole académico. 
Estudios Pedagógicos XLIV, $\mathrm{N}^{\circ}$ 2: 297-310, 2018

LA EVALUACIÓN FORMATIVA EN EL PROCESO ENSEÑANZA-APRENDIZAJE EN ESTUDIANTES DE ACTIVIDAD

DEPORTIVA DE LA UNIVERSIDAD DE COSTA RICA

Desde el punto de vista pedagógico, los resultados nos conducen a concluir que la inclusión de la práctica de actividad físico-deportiva en los planes curriculares puede potenciar el desarrollo integral de los futuros profesionales en los distintos saberes.

Como recomendación, se sugiere que la actividad deportiva pase a ser un curso anual y no semestral, con el fin de dar mayor oportunidad al estudiantado de participar de este tipo de cursos que potencien los distintos canales de crecimiento integral, físico, cognitivo, emocional y social. Así también, se recomienda ampliar la oferta curricular con contenidos $\mathrm{y}$ actividades alternativas, novedosas y actuales, como por ejemplo los deportes no tradicionales, entrenamientos de tipo funcional sugeridos por los mismos estudiantes del presente estudio.

\section{REFERENCIAS BIBLIOGRÁFICAS}

Arrieta, J., \& Díaz, L. (1989). Una visión humanista de los estudios generales a través de los seminarios participativos. Revista Estudios, 8, 35-42.

Asún-Dieste, S., Romero-Martín, R., \& Chivite-Izco, M. (2017). Exploración de sistemas de evaluación formativa entre estudiantes universitarios en la provincia de Huesca. Apunts: Educación física y deportes, 127, 52-58. doi:10.5672/apunts.2014-0983.es.(2017/1).127.05

Bain, K. (2007). Lo que hacen los mejores profesores de universidad. Valencia: Universidad de Valencia.

Casanova, M. A. (1998). Evaluación: Concepto, tipología y objetivos. La evaluación educativa. Escuela básica, 1, 67-102.

García, S. (1979). Evolución de la actividad deportiva de la Universidad de Costa Rica, desde 1957 hasta 1978 inclusive. San José: Universidad de Costa Rica.

González Peiteado, M., \& Pino Juste, M. (2014). Aproximación a las representaciones y creencias del alumnado de Magisterio sobre los estilos de enseñanza. Educación XX1, 17(1), 83-110. doi:10.5944/educxx1.17.1.10706

Hernández, R., Fernández, C., \& Baptista, P. (2006). Metodología de Investigación (4ta ed.). México: McGraw-Hill.

Hortigüela, D., Pérez-Pueyo, Á., \& López-Pastor, V. (2015). Implicación y regulación del trabajo del alumnado en los sistemas de evaluación formativa en educación superior. Revista electrónica de Investigación y Evaluación Educativa, 21(1), 1-15. doi:10.7203/relieve.21.1.5171

Martínez, A. C., Chillón, P., Martín-Matillas, M., Pérez, I., Castillo, R., Zapatera, B., . . . DelgadoFernández, M. (2012). Motivos de abandono y no práctica físico-deportiva en adolescentes españoles: estudio Avena. Cuadernos de Psicología del Deporte, 12(1), 45-54.

Renzi, G. M. (2009). Educación Física y su contribución al desarrollo integral de los niños en la primera infancia. Revista iberoamericana de educación, 50(7), 1-14.

Romero-Martín, R., Castejón-Oliva, F., \& López-Pastor, V. (2015). Divergencias del alumnado y del profesorado universitario sobre las dificultades para aplicar la evaluación formativa. Revista electrónica de Investigación y Evaluación Educativa, 21(1), 1-16. doi:10.7203/relieve.21.1.5169

Watson, A., Timperio, A., Brown, H., Best, K., \& Hesketh, K. D. (2017). Effect of classroom-based physical activity interventions on academic and physical activity outcomes: a systematic review and meta-analysis. International Journal of Behavioral Nutrition \& Physical Activity, 14, 1-24. doi:10.1186/s12966-017-0569-9

Wilson, F. R., Pan, W., \& Schumsky, D. A. (2012). Recalculation of the critical values for Lawshe's content validity ratio. Measurement and Evaluation in Counseling and Development, 45(3), 197-210. doi:10.1177/0748175612440286 